\title{
Efeito do Hexazinone Isolado e em Mistura na Eficiência FotossintétICA DE Panicum maximum
}

\author{
Effect of Hexazinone Applied Alone and in Combination on the Photosynthetic Efficiency of \\ Panicum maximum
}

\begin{abstract}
GIROTTO, M. ${ }^{2}$, ARALDI, R. ${ }^{2}$, VELINI, E.D. ${ }^{3}$ TRINDADE, M.L.B. ${ }^{4}$ e CARBONARI, C.A. ${ }^{4}$
RESUMO - Esta pesquisa teve como objetivo avaliar a velocidade e intensidade de ação do hexazinone isolado e em mistura com outros inibidores do fotossistema II, através da eficiência fotossintética de Panicum maximum em pós-emergência. O ensaio foi constituído de seis tratamentos: hexazinone $\left(250 \mathrm{~g} \mathrm{ha}^{-1}\right)$, tebuthiuron $\left(1,0 \mathrm{~kg} \mathrm{ha}^{-1}\right)$, hexazinone + tebuthiuron $\left(125 \mathrm{~g} \mathrm{ha}^{-1}+0,5 \mathrm{~kg} \mathrm{ha}^{-1}\right)$, diuron $\left(2.400 \mathrm{~g} \mathrm{ha}^{-1}\right)$, hexazinone + diuron $\left(125+1.200 \mathrm{~g} \mathrm{ha}^{-1}\right)$, metribuzin $\left(1.440 \mathrm{~g} \mathrm{ha}^{-1}\right)$, hexazinone + metribuzin $\left(125+720 \mathrm{~g} \mathrm{ha}^{-1}\right)$ e uma testemunha. $\mathrm{O}$ experimento foi instalado em delineamento inteiramente casualizado, com quatro repetições. Após a aplicação dos tratamentos, as plantas foram transportadas para casa de vegetação sob condições controladas de temperatura e umidade, onde ficaram durante o periodo experimental, sendo realizadas as seguintes avaliações: taxa de transporte de elétrons e análise visual de intoxicação. A avaliação com o fluorômetro foi realizada nos intervalos de $1,2,6,24,48,72,120$ e 168 horas após a aplicação, e as avaliações visuais, aos três e sete dias após a aplicação. Os resultados demonstraram diferença nos tratamentos, enfatizando a aplicação do diuron, que reduziu lentamente o transporte de elétrons comparado com os outros herbicidas e, em mistura com hexazinone, apresentou efeito sinérgico. Verificou-se com o uso do fluorômetro a intoxicação antecipada em plantas de P. maximum após a aplicação de herbicidas inibidores do fotossistema II de forma isolada e em mistura.
\end{abstract}

Palavras-chave: fluorescência, fluxo de elétrons, fotossistema, fluorômetro.

ABSTRACT - This work aimed to evaluate the speed and intensity of action of hexazinone applied alone and in combination with other photo-system II inhibitors on the photosynthetic efficiency of Panicum maximum in post-emergence. The assay consisted of six treatments: hexazinone (250 g ha-1), tebuthiuron (1.0 kg ha-1), hexazinone + tebuthiuron (125 g ha-1 $+0.5 \mathrm{~kg} \mathrm{ha-1})$, diuron $\left(2,400 \mathrm{~g} \mathrm{ha}^{-1}\right)$, hexazinone + diuron $\left(125+1,200 \mathrm{~g} \mathrm{ha}^{-1}\right)$, metribuzin $\left(1,440 \mathrm{~g} \mathrm{ha}^{-1}\right)$, hexazinone + metribuzin $\left(125+720 \mathrm{~g} \mathrm{ha}^{-1}\right)$ and a control. The experiment was conducted in a randomized design with four replications. After treatment application, plants were transported to a greenhouse with controlled temperature and humidity, remaining there throughout the experimental period, during which electron transport rate and visual analysis of intoxication were assessed. The fluorometer evaluation was performed at intervals of $01,02,06,24,48,72,120$, and 168 hours after application, and visual assessments at 3 and 7 days after application. The results showed differences in the treatments, with the application of the herbicide diuron slowly reducing electron transport, compared with the other herbicides, and presenting a synergistic effect when mixed with hexazinone. Early intoxication of $\boldsymbol{P}$. maximum plants was verified with the use of the fluorometer, after application of the photo-system II inhibitors, alone and in combination.

Keywords: fluorescence, electron flow, photo-system, fluorometer.

Recebido para publicação em 10.6.2011 e aprovado em 13.7.2012.

2 Pós-Graduação em Agronomia pela Faculdade de Ciências Agronômicas - FCA/UNESP - Campus de Botucatu, Fazenda Experimental Lageado, Caixa Postal 237, 18603-970 Botucatu-SP, <girotto@fca.unesp.br>; ${ }^{3}$ Professor, Dr., Dep. de Agricultura. FCA/UNESP Campus de Botucatu; ${ }^{4}$ Doutores, FCA/UNESP - Campus de Botucatu, Núcleo de Pesquisa Avançada em Matologia - NUPAM.

Planta Daninha, Viçosa-MG, v. 30, n. 2, p. 341-347, 2012 


\section{INTRODUÇÃO}

As espécies de $P$. maximum habitam espontaneamente as principais áreas de cultivos no Brasil. Seu alto grau de interferência no desenvolvimento das plantas cultivadas e a concorrência por água, luz e nutrientes do solo proporcionam redução na produção agrícola (Pitelli, 1985).

Na condição atual de produção, o método químico é o mais utilizado no controle de Panicum maximum e das demais plantas daninhas, em razão das extensas áreas cultivadas, escassez de mão de obra, facilidade de aplicação, custo e eficácia do tratamento, além de ser um método econômico e de alto rendimento em comparação com os outros (Freitas et al., 2004).

Em relação ao controle químico, é importante lembrar que há centenas de espécies de plantas daninhas, e estas podem apresentar diferentes características morfológicas e fisiológicas, que lhes conferem comportamento diferenciado em relação aos herbicidas utilizados. Além disso, a necessidade de diminuir os custos de produção da cultura tem levado os produtores a utilizar misturas de herbicidas (Silva et al., 2007).

As misturas de herbicidas geralmente são utilizadas com o intuito de aumentar o espectro de controle das plantas daninhas e também de reduzir as doses aplicadas, proporcionando menor toxicidade à cultura, menor efeito residual no solo e diminuição dos custos de produção (Kruse et al., 2000).

Há grande expansão no uso de misturas e na aplicação sequencial de herbicidas em um único ciclo das culturas, porém o manejo de herbicidas, especialmente das misturas, requer grande cuidado quanto a precauções para evitar contaminação do solo e água, além do conhecimento a respeito das interações entre os produtos, visando obter o máximo de controle de plantas daninhas com efeitos sinergísticos e minimizar injúrias às culturas (Silva et al., 2007).

Em trabalho realizado por Carvalho et al. (2010) com aplicação de mesotrione + ametryn foi observado efeito sinergístico entre os herbicidas, evidenciando, pelos dados de controle para as 10 espécies daninhas estudadas, que o efeito de mistura foi maior do que a eficácia de cada um dos herbicidas isoladamente.

Para controlar as principais plantas daninhas nos plantios de cana-de-açúcar, vários herbicidas com diferentes mecanismos de ação e formulações estão registrados para uso no Brasil. Entre os herbicidas, destacamse hexazinone, tebuthiuron, diuron e metribuzin. Esses produtos atuam inibindo a eficiência fotossintética das plantas, ligandose à proteína $D_{1}$, que é considerada o sítio de ligação da quinona $B\left(Q_{B}\right)$, a qual se localiza nas membranas dos tilacoides dos cloroplastos, causando, por consequência, o bloqueio do transporte de elétrons de $Q_{A}$ para $Q_{B}$. Assim, interrompe a fixação de $\mathrm{CO}_{2}$ e a produção de ATP e $\mathrm{NADPH}_{2}$, os quais são elementos essenciais para o crescimento das plantas (Breitenbach et al., 2001).

Em relação à fase de transporte de elétrons durante a fotossintese nas plantas, a luz é absorvida pela clorofila e, ao excitarem os elétrons, promovem a transferência da energia para os centros de reação dos fotossistemas II e I (Young \& Frank, 1996). Quando ocorre excesso de energia, esta pode ser dissipada na forma de fluorescência (Krause \& Winter, 1996). Portanto, uma das formas de monitoramento da inibição ou redução na transferência de elétrons entre os fotossistemas das plantas submetidas à aplicação de herbicidas, que pode ser observada ainda em folhas intactas, é a fluorescência da clorofila (Maxwell \& Johnson, 2000); a redução na dissipação da energia pelo processo fotoquímico é refletida por incremento correspondente na fluorescência.

A análise da fluorescência da clorofila vem sendo largamente utilizada no entendimento dos mecanismos da fotossintese propriamente dita, bem como na avaliação da capacidade fotossintética alterada com a aplicação de herbicidas (Ireland et al., 1986). Para esse tipo de avaliação são utilizados fluorômetros, em aplicações que variam desde a rápida identificação de injúrias causadas ao aparelho fotossintético, mesmo quando os sintomas não sejam considerados visíveis, até a análise detalhada da alteração da capacidade fotossintética da planta. 
A pesquisa teve como objetivo avaliar a velocidade e intensidade de ação do hexazinone isolado e em mistura com outros inibidores do fotossistema II, através da eficiência fotossintética em Panicum maximum em pós-emergência.

\section{MATERIAL E MÉTODOS}

O experimento foi conduzido em casa de vegetação sob ambiente controlado, e o local do experimento apresentou as seguintes coordenadas geográficas: latitude de $22^{\circ} 07^{\prime} 56^{\prime \prime}$ S, longitude de $74^{\circ} 66^{\prime} 84^{\prime \prime} \mathrm{W}$ e altitude de $762 \mathrm{~m}$.

O solo utilizado como substrato foi inicialmente seco à sombra por um período de 48 horas. Depois disso, foi passado em peneira com malha de 200 mesh, sendo submetido às análises químicas e granulométricas (Tabela 1). Com base nesses resultados, o solo foi adubado para propiciar melhor crescimento das plantas daninhas e, em seguida, acondicionado em vasos de $2 \mathrm{dm}^{3}$ de capacidade, procedendo-se à semeadura das sementes de $P$. maximum.

O ensaio foi constituído de sete tratamentos: hexazinone (250 $\left.\mathrm{g} \mathrm{ha}^{-1}\right)$, tebuthiuron $\left(1,0 \mathrm{~kg} \mathrm{ha}{ }^{1}\right)$, hexazinone + tebuthiuron (125 $\left.\mathrm{g} \mathrm{ha}^{-1}+0,5 \mathrm{~kg} \mathrm{ha}^{-1}\right)$, diuron (2.400 $\left.\mathrm{g} \mathrm{ha}^{-1}\right)$, hexazinone + diuron $\left(125+1.200 \mathrm{~g} \mathrm{ha}^{-1}\right)$, metribuzin $\left(1.440 \mathrm{~g} \mathrm{ha}^{-1}\right)$, hexazinone + metribuzin $\left(125+720 \mathrm{~g} \mathrm{ha}^{-1}\right)$ e uma testemunha. O experimento foi instalado em delineamento inteiramente casualizado, com quatro repetições.

A aplicação dos herbicidas foi realizada utilizando um pulverizador estacionário instalado em laboratório e munido de uma barra contendo quatro pontas do tipo XR11002. A pulverização foi feita sob pressão constante de 1,5 bar, pressurizado por ar comprimido, com consumo de calda de $200 \mathrm{~L} \mathrm{ha}^{-1}$. A temperatura no momento da aplicação era de $26{ }^{\circ} \mathrm{C}$, com umidade relativa de $73 \%$.

Após aplicação dos herbicidas, as plantas foram transportadas para casa de vegetação sob condições controladas de temperatura e umidade, onde ficaram até o término do ensaio (sete dias após aplicação), sendo realizadas as seguintes avaliações: taxa de transporte de elétrons e análise visual de intoxicação. A avaliação dos tratamentos foi realizada visualmente aos 3 e 7 dias após a aplicação (DAA) dos herbicidas, através de uma escala de notas em porcentagem, em que zero correspondia a nenhuma injúria e 100 à morte de plantas (SBCPD, 1995).

Após a aplicação dos herbicidas, foi registrada a leitura da taxa de transporte de elétrons (ETR) na porção mediana das folhas de $P$. maximum, com um fluorômetro portátil, modelo Multi-Mode Chlorophyll Fluorometer OS5p (Opti-Sciences); os intervalos utilizados para avaliação foram de 1, 2, 6, 24, 48, 72, 120 e 168 horas após a aplicação.

A fluorescência no aparelho foi a fonte diodo com pico de luz vermelha no comprimento de onda de $660 \mathrm{~nm}$, sendo bloqueadas radiações maiores que $690 \mathrm{~nm}$. A intensidade média da luz foi ajustada para o intervalo de 0 a $1 \mu \mathrm{Mol} \mathrm{m}^{-2} \mathbf{s}^{-1}$, com o uso da lâmpada halogênica de $35 \mathrm{~W}$. O feixe de luz foi opticamente monitorado no interior da câmara, para corrigir no aparelho as variações devidas às mudanças na temperatura do ambiente no aparelho. Os

Tabela 1 - Análises química e granulométrica do solo cultivado com P. maximum-Botucatu-SP-2011

\begin{tabular}{|c|c|c|c|c|c|c|c|c|c|c|}
\hline \multirow{2}{*}{ Solo } & \multirow{2}{*}{$\begin{array}{c}\mathrm{pH} \\
\left(\mathrm{CaCl}_{2}\right) \\
\end{array}$} & \multirow{2}{*}{$\begin{array}{c}\mathrm{MO} \\
\left(\mathrm{g} \mathrm{dm}^{-3}\right) \\
\end{array}$} & \multirow{2}{*}{$\begin{array}{c}\text { P res. } \\
\left(\mathrm{mg} \mathrm{dm}^{-3}\right) \\
\end{array}$} & $\mathrm{K}^{+}$ & $\mathrm{Ca}^{+2}$ & $\mathrm{Mg}^{+2}$ & $\mathrm{H}^{+}+\mathrm{Al}^{+3}$ & SB & $\mathrm{T}$ & $\mathrm{V}(\%)$ \\
\hline & & & & \multicolumn{6}{|c|}{$\left(\mathrm{mmol}_{\mathrm{c}} \mathrm{dm}^{3}\right)$} & \\
\hline LVd & 4,3 & 19 & 1 & 0,6 & 10 & 4 & 58 & 14,6 & 73 & 21 \\
\hline \multirow{3}{*}{\multicolumn{2}{|c|}{ Granulometria (\%) }} & \multicolumn{9}{|c|}{ Classe de solo } \\
\hline & & \multirow{2}{*}{ Argila } & \multirow{2}{*}{ Limo } & \multicolumn{6}{|c|}{ Areia } & \multirow{2}{*}{$\begin{array}{l}\text { Classe } \\
\text { textura }\end{array}$} \\
\hline & & & & Fin & & Média & Grossa & & Total & \\
\hline \multicolumn{2}{|c|}{ LVd } & 20 & 4 & 22 & & 35,7 & 17,4 & & 7,6 & Média \\
\hline
\end{tabular}

Departamento de Solos - FCA/UNESP - Botucatu. 
sinais ópticos foram transferidos para a superficie da folha por uma trifurcação personalizada de fibra óptica, sendo de $2 \mathrm{~cm}^{2}$ a área iluminada utilizando protocolo Yield, que é otimizado para um estado da fotossintese adaptado à luz porque registra as medidas de quantum efetivo produzido no fotossistema II (PSII). Tanto a fonte de luz do sol quanto a luz artificial podem ser usadas para dirigir a fotossintese. A luz reemitida foi conduzida via fibra óptica para o aparelho por meio de três conectores ligados à lateral do OS5p. As análises foram feitas conforme a metodologia de Genty et al. (1989), avaliando a emissão da fluorescência da clorofila na superficie superior das folhas.

A taxa de transporte de elétrons dada em $\mu$ Mols elétrons $\mathrm{m}^{-2} \mathbf{s}^{-1}=(\mathrm{Y}) \cdot(\mathrm{PAR}) \cdot(0,84) \cdot(0,5)$ é equivalente a: (produção de quantum do PSII) $\mathrm{x}$ (medidas da radiação fotossinteticamente ativa medida em $\mu$ Mols elétrons $\left.\mathrm{m}^{-2} \mathrm{~s}^{-1}\right) \mathrm{x}$ (coeficiente de absorção da folha) $\mathrm{x}$ (fração de luz absorvida pelo complexo antena do PSII). O ETR é uma medida da separação de cargas do centro de reação do PSII. Na equação são usados valores-padrão, porém ambos os coeficientes de absorção e fração da luz absorvida pelo PSII podem ser trocados (Laisk \& Loreto, 1996).

Como o parâmetro ETR determina a taxa de transporte de elétrons fotossintéticos no PSII, o uso do valor do ETR permite detectar o efeito da atuação do herbicida em nivel de concentração de 0,5 micromoles. $\mathrm{dm}^{-3}$, enquanto o método tradicional, que inclui a medição do parâmetro $\mathrm{Fv} / \mathrm{Fm}$, permite detectar apenas em um nivel de concentração que é 100 vezes maior (Korres et al., 2003; Abbaspor et al., 2006), justificando o uso da metodologia empregada.

Os dados da taxa de transporte de elétrons (ETR) foram expressos em porcentagem em relação à testemunha e submetidos à análise de regressão. A equação hiperbólica racional utilizada para ajustar os dados de ETR em relação ao tempo para os tratamentos é descrita a seguir:

$$
y=\frac{1}{a+(b x)}
$$

em que $y$ representa o ETR, $x$ representa o tempo e $a$ e $b$ são coeficientes de ajuste da equação. Para o ajuste do comportamento do hexazinone foi utilizada a equação hiperbólica racional com três coeficientes: $a, b$ e $c$.

Quanto aos dados da intoxicação visual, foi realizada análise de variância, sendo as médias comparadas pelo teste $\mathrm{t}$ a $10 \%$ de significância (Velini et al., 2006).

\section{RESULTADOS E DISCUSSÃO}

Nas Figuras 1, 2 e 3 são representadas as curvas obtidas para os herbicidas aplicados sobre $P$. maximum; na Tabela 2 são apresentados os resultados das análises de regressão entre os valores de ETR e os intervalos de tempo, segundo o modelo apresentado.

Pela análise da Figura 1, observa-se que o herbicida tebuthiuron apresentou inicialmente menor efeito de redução do ETR comparado com o hexazinone, que foi o tratamento aplicado de forma isolada mais efetivo em inibir o fotossistema de $P$. maximum. A mistura de hexazinone e tebuthiuron não mostrou diferença em relação ao herbicida hexazinone aplicado isoladamente - ambos apresentaram bom controle. Em trabalho de Carvalho et al. (2010), a aplicação da mistura de mesotrione + ametryn também proporcionou eficiente controle em P. maximum.

Observa-se, na Figura 2, que o herbicida diuron reduziu lentamente o transporte de

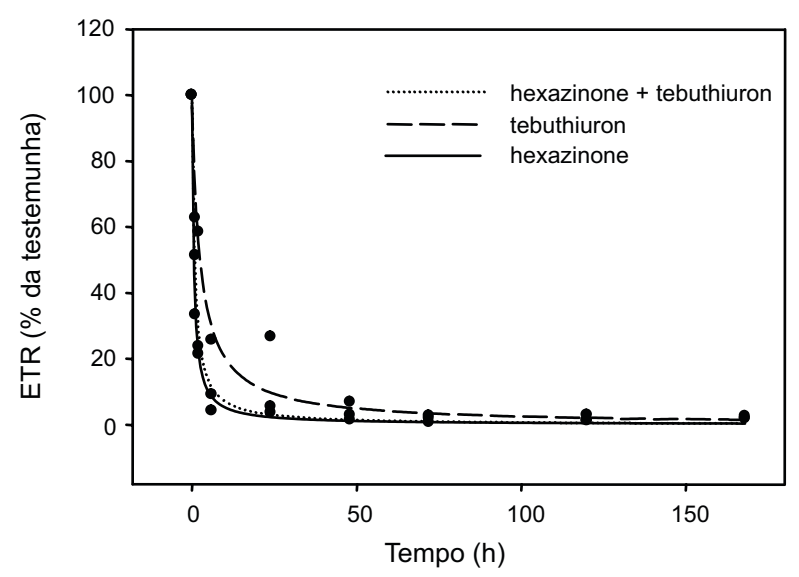

Figura 1 - Efeito do hexazinone e tebuthiuron isolados e em mistura sobre a taxa de transporte de elétrons ( $\mu \mathrm{mol}$ elétrons $\mathrm{m}^{-2} \mathrm{~s}^{-1}$ ) ao longo do tempo (horas). Botucatu-SP, 2011. 


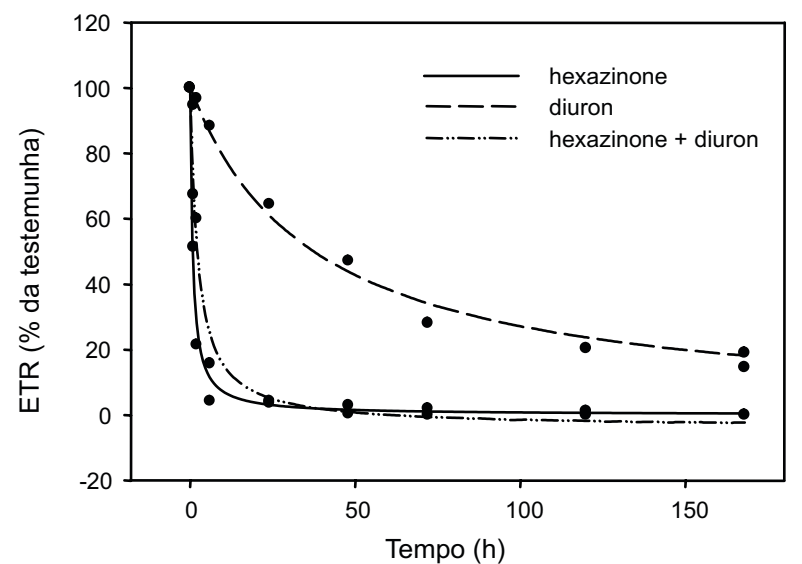

Figura 2 - Efeito do hexazinone isolado e em mistura com o diuron sobre a taxa de transporte de elétrons ( $\mu \mathrm{mol}$ elétrons $\mathrm{m}^{-2} \mathrm{~s}^{-1}$ ) ao longo do tempo (horas). Botucatu-SP, 2011.

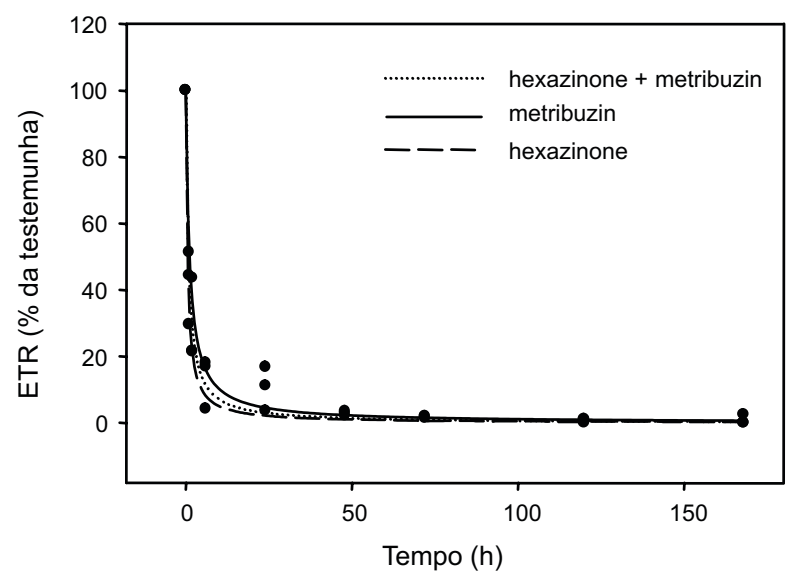

Figura 3 - Efeito do hexazinone isolado e em mistura com o metribuzin sobre a taxa de transporte de elétrons ( $\mu \mathrm{mol}$ elétrons $\mathrm{m}^{-2} \mathrm{~s}^{-1}$ ) ao longo do tempo (horas). Botucatu-SP 2011. elétrons em $P$. maximum quando comparado com o hexazinone, o qual apresentou rápida redução do ETR. Enquanto o hexazinone gastou seis horas após a aplicação para a inibição completa do ETR, o diuron, mesmo após 168 horas do início das avaliações, não havia reduzido ainda completamente o ETR. Em trabalho desenvolvido por Dayan et al. (2009), foi monitorado o ETR em Digitaria sangrinalis e Abutilon theophrash, quando submetidas à aplicação de amicarbazone e atrazine, ambos inibidores do PSII, sendo o transporte de elétrons completamente inibido com oito horas após a aplicação dos tratamentos nas plantas.

Na mistura de diuron e hexazinone, observa-se ainda que não houve muita diferença no comportamento dos tratamentos de hexazinone + diuron e hexazinone isolado, evidenciando efeito sinergístico da mistura. O tempo necessário para redução de 50\% do ETR foi de 36 horas para as plantas submetidas ao diuron e de uma e duas horas para as plantas aplicadas com hexazinone e hexazinone + diuron, respectivamente.

Em trabalho de Girotto et al. (2010) foram observados para Brachiaria decumbens 30\% de redução do ETR duas horas após a aplicação do diuron, e com 24 horas foi obtida a inibição do transporte de elétrons, atuando assim, de forma mais rápida, o herbicida quando aplicado em B. decumbens, em comparação com $P$. maximum.

Nos tratamentos em que foram aplicados hexazinone e metribuzin de forma isolada e em mistura em $P$. maximum, não foi verificada diferença no comportamento dos dados que

Tabela 2 - Parâmetros da regressão e coeficiente de determinação de modelos exponenciais ajustados

\begin{tabular}{|l|c|c|c|c|}
\hline \multirow{2}{*}{ Herbicida } & \multicolumn{2}{|c|}{ Modelo Hiperbólico Racional } & \multirow{2}{*}{$\mathrm{R}^{2}$} \\
\cline { 2 - 5 } & $\mathrm{A}$ & $\mathrm{B}$ & $\mathrm{C}$ & - \\
\hline Testemunha & - & - & - & 0.9825 \\
\hline Hexazinone & 0,0099 & 0,0130 & - & 0,9593 \\
\hline Tebuthiuron & 0,0103 & 0,0039 & - & 0,9971 \\
\hline Hexaz. + tebuth. & 0,0010 & 0,0181 & - & 0.9907 \\
\hline Diuron & 0,0099 & 0,0003 & - & 0,9859 \\
\hline *Hexaz. + diur. & 101,0498 & $-1,6517$ & 0,4471 & 0,9799 \\
\hline Metribuzin & 0,0101 & 0,0087 & - & 0,9568 \\
\hline Hexaz. + metrib. & 0,0101 & 0,0187 & - & 2 \\
\hline
\end{tabular}

* Modelo hiperbólico racional com três parâmetros. 
relacionam ETR, em relação ao intervalo de tempo (Figura 3); uma hora após a aplicação dos herbicidas, as plantas já apresentavam mais de $50 \%$ de redução do ETR.

Na Figura 4 estão apresentados os resultados referentes às médias das avaliações de intoxicação aos 3 DAA. Nessa primeira avaliação, verificaram-se dois comportamentos diferentes de intoxicação: os tratamentos de tebuthiuron + hexazinone, diuron + hexazinone e metribuzin, que apresentaram entre 20 e $25 \%$ de controle visual, e metribuzin + hexazinone, tebuthiuron e diuron, que mostraram menor porcentagem de intoxicação, com valores próximos a $5 \%$ de controle apenas.

A Figura 5 demonstra que aos 7 DAA houve maior porcentagem de controle para os tratamentos em estudo. Os herbicidas de maior controle na primeira avaliação também o mantiveram aos 7 DAA, sendo em torno de $80 \%$ de intoxicação para as plantas de $P$. maximum, comparado com os demais herbicidas. O diuron apresentou menor porcentagem de controle, com valores próximos a $20 \%$.

Segundo Fornarolli et al. (1997), com o uso dos herbicidas diuron + MSMA houve eficiente controle de plantas daninhas em pósemergência. A aplicação de metribuzin juntamente com o mesotrione proporcionou $81,3 \%$ de controle para $P$. maximum e $B$. plantaginea (Carvalho et al., 2010).

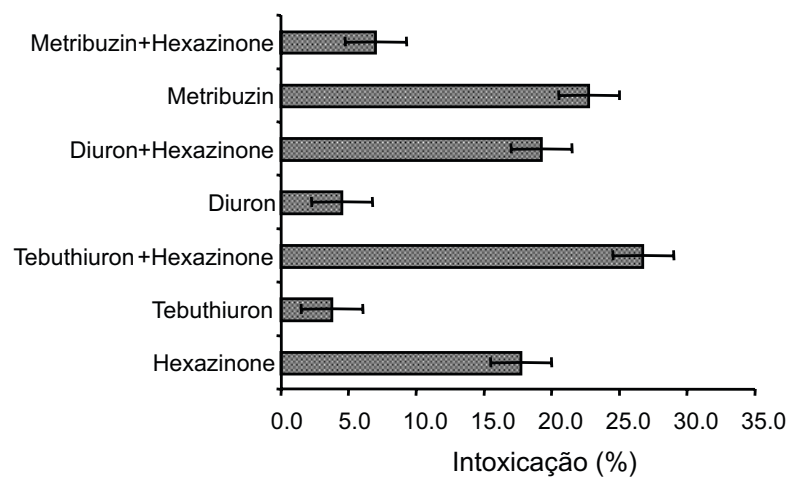

Figura 4 - Porcentagem de intoxicação visual verificada para $P$. maximum após três dias da aplicação de hexazinone, tebuthiuron, hexazinone + tebuthiuron, diuron, hexazinone + diuron, metribuzin e hexazinone + metribuzin. Barras no gráfico indicam valores de DMS. Botucatu-SP-2011.

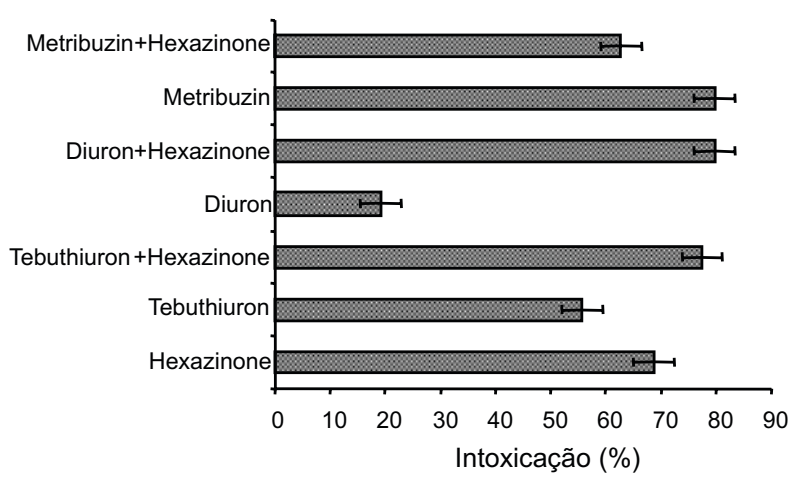

Figura 5 - Porcentagem de intoxicação visual verificada para P. maximum após sete dias da aplicação de hexazinone, tebuthiuron, hexazinone + tebuthiuron, diuron, hexazinone + diuron, metribuzin e hexazinone + metribuzin. Barras no gráfico indicam valores de DMS. Botucatu-SP-2011.

Conforme o experimento realizado, observou-se diferença nos tratamentos, enfatizando que a aplicação do diuron apresentou efeito sinérgico quando em mistura com o hexazinone. Com o uso do fluorômetro, é possível verificar a intoxicação antecipada em plantas de $P$. maximum após a aplicação de herbicidas inibidores do fotossistema II de forma isolada e em mistura.

\section{LITERATURA CITADA}

ABBASPOOR, M.; TEICHER H. B.; STREIBIG. J. C. The effect of root-absorbed PSII inhibitors on Kautsky curve parameters in sugar beet. Weed Res. v. 46, n. 3, p. 226-235, 2006.

BREITENBACH, J.; ZHU, C.; SANDMAN, G. Bleaching herbicide norflurazon inhibits phytoene desaturase by competition with the cofactors. J. Agric. Food Chem., v. 49, n. 11, p. $5270-5272,2001$.

CARVALHO, F. T. et al. Controle de dez espécies daninhas em cana-de-açúcar com o herbicida mesotrione em mistura com ametryn e metribuzin. Planta Daninha, v. 28, n. 3, p. $585-590,2010$.

DAYAN, F. E.; TRINDADE, M. L. B.; VELINI, E. D. Amicarbazone, a new photosystem II inhibitor. Weed Sci., v. 57, n. 6 , p. $579-583,2009$.

FORNAROLLI, D. A. et al. Controle do Sorghum arundinaceum em pós-emergência inicial e tardia com uso de MSMA + diuron na cultura da cana-de-açúcar. In: CONGRESSO BRASILEIRO DA CIÊNCIA DAS PLANTAS DANINHAS, 21., 1997, Caxambu, MG.

Resumos... Viçosa MG: Sociedade Brasileira de Planta Daninha, 1997. p. 259. 
FREITAS, S. P. et al. Controle químico de Rottboelia exaltata em cana-de-açúcar. Planta Daninha, v. 22, n. 3, p. 461-466, 2004.

GENTY, B.; BRIANTAIS, J. M.; BAKER, N. R. The relationship between the quantum yield of photosynthetic electron-transport and quenching of chlorophyll fluorescence.

Biochim. Biophys. Acta, v. 990, n. 1, p. 87-92, 1989.

GIROTTO, M. et al. Monitoramento da taxa de transporte de elétrons no fotossistema II em três espécies de plantas daninhas após a aplicação de diuron e isoxaflutole. In: CONGRESSO BRASILEIRO DA CIÊNCIA DAS PLANTAS DANINHAS, 27., 2010, Ribeirão Preto, SP. Anais... Viçosa MG: Sociedade Brasileira de Planta Daninha, 2010. p. 3086-3090.

IRELAND, C. R.; PERCIVAL, M. P.; BAKER, N. R. Modification of the induction of photosynthesis in wheat by glyphosate, an inhibitor of amino acid metabolism. J. Exper. Bot., v. 37, n. 176, p. 299-308, 1986.

KORRES, N. E., FROUD-WILLIAMS, R. J, MOSS, S. R. Chlorophyll fluorescence technique as a rapid diagnostic test of the effects of the photosynthetic inhibitor chlortoluron on two winter wheat cultivars. Ann. Appl. Biol., v. 143, n. 6, p. 53-56, 2003.

KRAUSE, G. H.; WINTER, K. Photoinhibition of photosynthesis in plants growing in natural tropical forest gaps: a chlorophyll fluorescence study. Bot. Acta, v. 109, n. 6 , p. 456-462, 1996.

KRUSE, N. D.; TREZZI, M. M.; VIDAL, R. A. Herbicidas inibidores do EPSPS: Revisão de literatura. R. Bras. Herb., v. 1, n. 2, p. 139-46, 2000.
LAISK, A.; LORETO, F. Determining photosynthetic parameters from leaf $\mathrm{CO}_{2}$ exchange and chlorophyll fluorescence. Ribulose-1,5-bisphosphate carboxylase/ oxygenase specificity factor, dark respiration in the light Plant Physiol, v. 110, n. 3, p. 903-912, 1996.

MAXWELL, K; JOHNSON, G. N. Chlorophyll fluorescence: a practical guide. J. Exper. Bot., v. 51, n. 345, p. $659-668,2000$.

PITELLI, R. A. Interferência de plantas daninhas em culturas agrícolas. Inf. Agropec., v. 11, n. 129, p. 16-27, 1985.

SILVA, J. F. et al. Herbicidas: absorção, translocação, metabolismo, formulação e misturas. In: SILVA, A. A.; SILVA, J. F. Tópicos em manejo de plantas daninhas. Viçosa, MG: Universidade Federal de Viçosa, 2007. 367 p.

SOCIEDADE BRASILEIRA DA CIÊNCIA DAS PLANTAS DANINHAS - SBCPD. Procedimentos para instalação, avaliação e análise de experimentos com herbicidas Londrina: 1995. $42 \mathrm{p}$.

VELINI, E. D. et al. Interferência de plantas daninhas na cultura do milho. I - Efeito do número de repetições sobre a precisão dos resultados obtidos. Planta Daninha, v. 24, n. 3 , p. 435-442, 2006.

YOUNG, A. L.; FRANK, H. A. Energy transfer reactions involving carotenoids: quenching of chlorophyll fluorescence. J. Photochem. Photobiol. B: Biol., v. 36, n. 1, p. 3-15, 1996. 